\title{
Malignant obstructive jaundice - brachytherapy as a tool for palliation
}

\author{
Sandeep Jain, MD', Tejinder Kataria, MD', Shyam Singh Bisht, MD', Deepak Gupta, MD', Subramani Vikraman, MSc', \\ Sanjay Baijal, MD², Randhir Sud, MD³ \\ IMedanta Cancer Institute, Medanta - The Medicity, 2Division of Intervention Radiology, Medanta - The Medicity, ${ }^{3}$ Institute of Gastroenterology, \\ Medanta - The Medicity, Gurgaon Haryana, India
}

\begin{abstract}
Purpose: Malignant obstructive jaundice (MOJ) is relieved by stenting via endoscopic retrograde cholangiopancreatography (ERCP) or percutaneous transhepatic cholangiography and biliary drainage (PTCD). Stent occlusion rates of $30-45 \%$ have been reported in literature due to tumor ingrowth or overgrowth. We prospectively evaluated the feasibility and the role of intraluminal brachytherapy (ILBT) in preventing stent blockage in patients with MOJ after PTCD and stenting.

Material and methods: Twelve patients with MOJ who underwent PTCD followed by self expanding metallic stent (SEMS) placement were prospectively enrolled in this study. Written informed consent was obtained. Intraluminal brachytherapy was done once patient was stable and serum bilirubin was less than $2 \mathrm{mg} \%$ or $50 \%$ of baseline value. On the day of ILBT, 6 French brachytherapy catheters were placed across malignant stricture under fluoroscopic guidance with placement of the tip $1 \mathrm{~cm}$ distal to stricture. A dose of 10 to $14 \mathrm{~Gy}$ was delivered at $1 \mathrm{~cm}$ from central axis of the source. Suitable patients also received external beam radiotherapy (EBRT) with weekly concurrent chemotherapy.

Results: All patients tolerated the procedure well with minimal acute and late toxicities. Duodenal ulceration was observed in 1 patient. At a mean follow up of 10.25 months (5-24 months), stents were patent in 10/12 subjects and stent patency duration of 9.8 months (5-22) was reported.

Conclusions: Intraluminal brachytherapy post PTCD is feasible and effective in preventing stent occlusion with minimal acute and late toxicities.

Key words: intraluminal brachytherapy, malignant obstructive jaundice.

\section{Purpose}

Malignant obstructive jaundice (MOJ) can be caused by cholangiocarcinoma, gallbladder carcinoma, metastatic lymphadenopathy and pancreatic carcinoma. Most of the cases are inoperable at diagnosis and are treated with palliative intent. Relieving the obstruction and normalizing the serum bilirubin level is usually the first step in this endeavor. Cholangiopancreatography (ERCP) and stenting is the preferred method for relieving the obstruction. When obstruction is localized in the intrahepatic radicals and ERCP is not possible, percutaneous transhepatic cholangiography and biliary drainage (PTCD) is the only feasible option. Biliary decompression and stenting is effective in relieving the jaundice, however, high rates of stent occlusion (30-45\%) has been reported. Cholangiocarcinoma and gallbladder carcinoma have a tendency to invade bile ducts, hence stent occlusion due to tumor ingrowth or overgrowth is inevitably high [1,2]. Thus, in addition to stenting, an effective therapy is needed which can provide high palliation in form of prolong stent patency. External beam radiotherapy (EBRT) has been attempted, but major deterrents to improve results with EBRT alone are proximity of adjoining critical normal structures, high doses required to effectively irradiate these neoplasms, lack of clear localization of lesion and respiratory movements [3-5]. Many of these issues can be addressed with newer techniques like image guided radiotherapy, gated or active breathing coordinator guided radiotherapy or stereotactic body radiotherapy. Intraluminal brachytherapy (ILBT) alone or in combination with EBRT can be very useful, especially with its special dosimetric characteristics, accurate localization and short duration of treatment. Intraluminal brachytherapy could provide useful palliation and possibly improve survival by preventing stent re-occlusion, and tumor control [6-9].

The purpose of this pilot study was to assess the feasibility of high dose rate intraluminal brachytherapy (HDRILBT) and to evaluate the role of HDR-ILBT in preventing stent blockage in patients with MOJ after PTCD. 
Table 1. Patient characteristics

\begin{tabular}{lc} 
Mean age & 59 years (42-73) \\
\hline Number of patients & 12 \\
\cline { 2 - 2 } Male & 8 \\
\hline Female & 4 \\
\hline ECOG score & 4 \\
\hline 0 & 6 \\
\hline 1 & 2 \\
\hline 2 & 7 \\
\hline Histopathology & 5 \\
\hline Cholangiocarcinoma & $13.6(6-23)$ \\
\hline Gall bladder carcinoma
\end{tabular}

ECOG - Eastern Cooperative Oncology Group, PTCD - percutaneous transhepatic cholangiography and biliary drainage

\section{Material and methods}

A prospective pilot study was carried out at our institute between January 2011 and September 2012, in collaboration with division of intervention radiology and division of gastroenterology. Twelve consecutive inoperable patients of $\mathrm{MOJ}$ that were referred for PTCD and stenting were enrolled in the study after written informed consent. Table 1 shows patient characteristics. Most of these patients were in 50 to 70 years age group. Seven patients were diagnosed with cholangiocarcinoma and 5 had gall bladder carcinoma. Mean bilirubin level at the time of presentation was $13.6 \mathrm{mg} / \mathrm{dl}$. All patients underwent PTCD followed by HDR-ILBT successfully, without any per-operative events. Treatment characteristics is present in Table 2. Mean dose of 11.7 Gy (ranged 10 to $14 \mathrm{~Gy}$ ) was delivered at $1 \mathrm{~cm}$ from the central axis of source. Six of these patients received image guided EBRT (45 Gy in 25 fractions) after ILBT. Four patients also received weekly concurrent chemotherapy (5-FU and leucovorin) along with EBRT. Three patients were treated with palliative adjuvant chemotherapy (gemcitabine and oxaliplatin) after completion of ILBT. One patient developed two hepatic metastasis for which he received stereotactic body radiotherapy (SBRT) to a dose of 45 Gy in 3 fractions.

Percutaneous transhepatic cholangiography and biliary drainage and stenting were performed by intervention radiologist. Patients were eligible for ILBT once the serum bilirubin level was less than $2 \mathrm{mg} / \mathrm{dl}$ or $50 \%$ of baseline value after the intervention (PTCD followed by SEMS placement). Other inclusion criteria were ECOG performance status $0-2$, afebrile with no signs of cholangitis, hemodynamically stable, normal bleeding/clotting parameters, no evidence of distant metastasis and expected life expectancy of more than 6 months. Patients of end-stage malignant obstructive jaundice were not included in the study. Patients with serum total bilirubin concentration more than $50 \%$ of baseline value and features of cholangitis were kept under observation and medical management. They were taken up for ILBT once they fulfill the eligibility criteria.
Table 2. Treatment characteristics

\begin{tabular}{lc} 
Total number of catheters & 28 \\
\hline \begin{tabular}{l} 
Location of stricture \\
\hline Right hepatic duct
\end{tabular} \\
\hline Left hepatic duct & 7 \\
\hline Common hepatic duct & 12 \\
\hline Average length of stricture & $3.2 \mathrm{~cm}$ \\
\hline Dose prescribed & 11.67 Gy (10-14 Gy) \\
\hline Treatment & 6 \\
\hline EBRT & 1 \\
\hline SBRT & 5
\end{tabular}

EBRT - external beam radiation therapy, SBRT - stereotactic body radiation therapy

\section{Intraluminal brachytherapy procedure}

Intraluminal brachytherapy catheter placement, planning and radiation delivery were performed on the same sitting. Patients were taken on fluoroscopic couch and strictures were located with the help of contrast or air balloon. 10 French (F) external-internal drainage catheters which had been previously placed for bile drainage at the time of PTCD procedure was replaced by $7 \mathrm{~F}$ long vascular sheath. $6 \mathrm{~F}$ brachytherapy catheters (Lumencath ${ }^{\circledR}$, Nucletron, an Elekta company, Elekta AB, Stockholm, Sweden) with dummy sources were placed through the vascular sheath under fluoroscopic guidance, and the distal tip was placed $1 \mathrm{~cm}$ across the stricture. Attempts were made to place radiation catheters across all strictures. Patients were then shifted to integrated brachytherapy unit (IBU) for planning, evaluation and radiation delivery. Integrated brachytherapy unit has the advantage of carrying out imaging, planning and treatment on the same table without shifting the patient. In IBU orthogonal images were taken with radio-opaque dummies inside the brachytherapy catheters. Digital reconstruction of the catheters were done on the orthogonal images. Irradiated volume was customized to include $1 \mathrm{~cm}$ proximal, distal and radial to length of the stricture as determined by cholangiography (Fig. 1). A dose of 10 to 14 Gy was prescribed at $1 \mathrm{~cm}$ from central axis of the source. Dose optimization was done using Oncentra TPS Version 3.3 ${ }^{\circledR}(\mathrm{Nu}-$ cletron, an Elekta company, Elekta AB, Stockholm, Sweden). 3D dose distribution was obtained, evaluated and verified by SJ/TK (Fig. 2). Once the plan was approved, treatment was delivered by HDR Microselectron ${ }^{\circledR}$ (Nucletron, an Elekta company, Elekta AB, Stockholm, Sweden). External beam radiotherapy to a dose of $45 \mathrm{~Gy}$ in 25 fractions was delivered, 3 weeks after ILBT in suitable patients, in good condition. All patients were kept on three monthly follow up with liver function test. Symptoms were also recorded on every follow up. Six monthly computed tomography scan was also done for willing patients. Stent patency duration was evaluated for all patients. It was defined as duration 

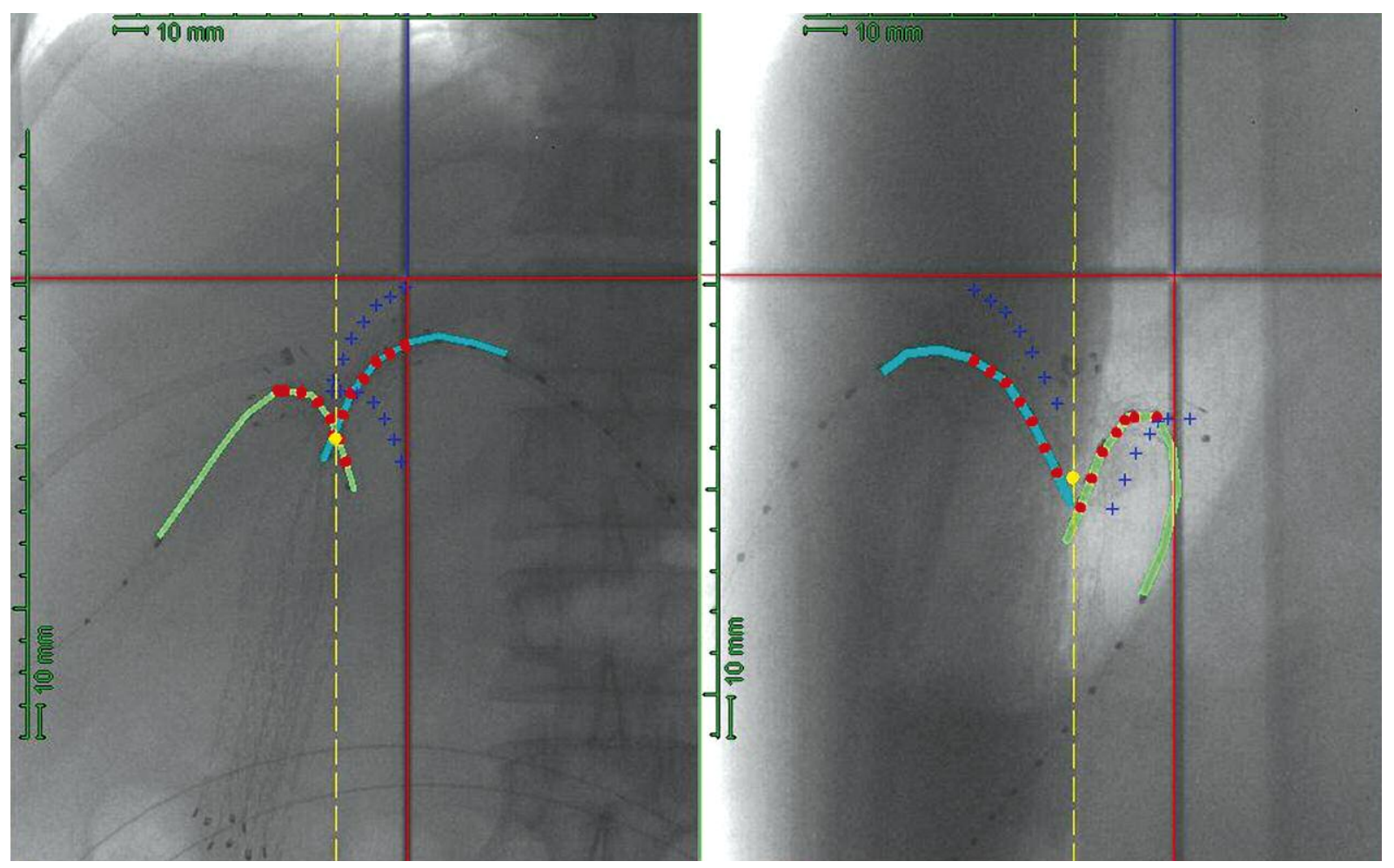

Fig. 1. Digital reconstruction of catheters and dose prescription $-1 \mathrm{~cm}$ from central axis

between PTBD and detection of elevated bilirubin level ( $>50 \%$ of nadir value) after ILBT. For patients who died or were lost to follow up before being diagnosed with stent re-occlusion, the date of last follow up was considered for assessing stent patency duration.

\section{Results}

Acute toxicities during and after ILBT treatment $(<30$ days) are presented in Table 3. All patients tolerated the treatment well. One third of patients had mild nausea and vomiting which was well controlled with antiemetics. Since all patients received prophylactic $3^{\text {rd }}$ generation cephalosporins before and after ILBT hence cholangitis, fever and sepsis were not seen as a treatment complication in any of these patients. At a mean follow-up of 10.3 months (ranged 5-24 months), five patients were alive with stable disease, one was alive with liver metastasis for which he received stereotactic body radiation therapy (SBRT) (Table 4). Five

Table 3. Acute toxicity profile

\begin{tabular}{lc} 
Toxicity & Incidence (\%) \\
\hline Nausea and vomiting (Gr I/II) & $4 / 12(33)$ \\
\hline Local site pain & $12 / 12(100)$ \\
\hline Cholangitis & $0 / 12(0)$ \\
\hline Bleeding & $0 / 12(0)$ \\
\hline Duodenal ulcers & $1 / 12(8.3)$
\end{tabular}

EBRT - external beam radiation therapy, SBRT - stereotactic body radiation therapy

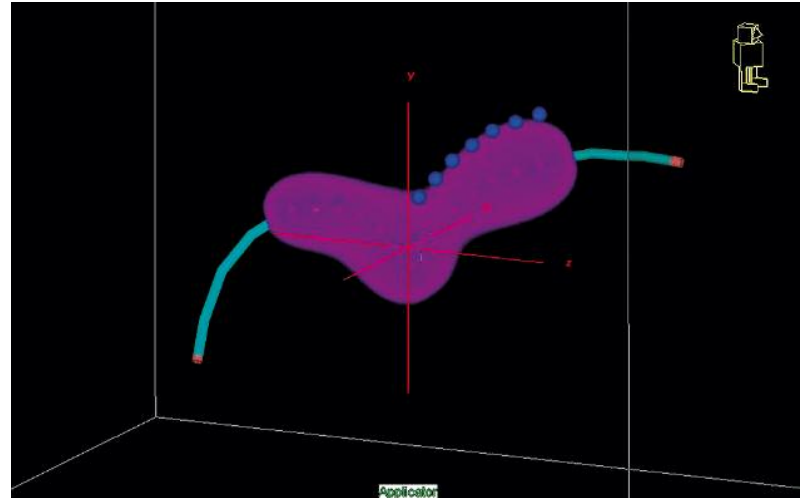

Fig. 2. Dose cloud: optimization and verification

Table 4. Results

\begin{tabular}{ll} 
Mean follow-up duration & 10.3 months (5-24 months) \\
\hline Alive with stable disease & 5 patients \\
\hline Alive with metastasis & 1 patient \\
\hline Dead with progressive disease & 5 patients \\
\hline Lost to follow-up & 1 patient \\
\hline Stent patency at last follow-up & $10 / 12$ (83\%) patients \\
\hline Stent patency duration (all) & 9.8 months (5-22 months) \\
\hline $\begin{array}{l}\text { Stent patency duration } \\
\text { (ILBT + EBRT) }\end{array}$ & 12.8 months (7-22 months) \\
\hline
\end{tabular}

ILBT - intraluminal brachytherapy, EBRT - external beam radiation therapy 


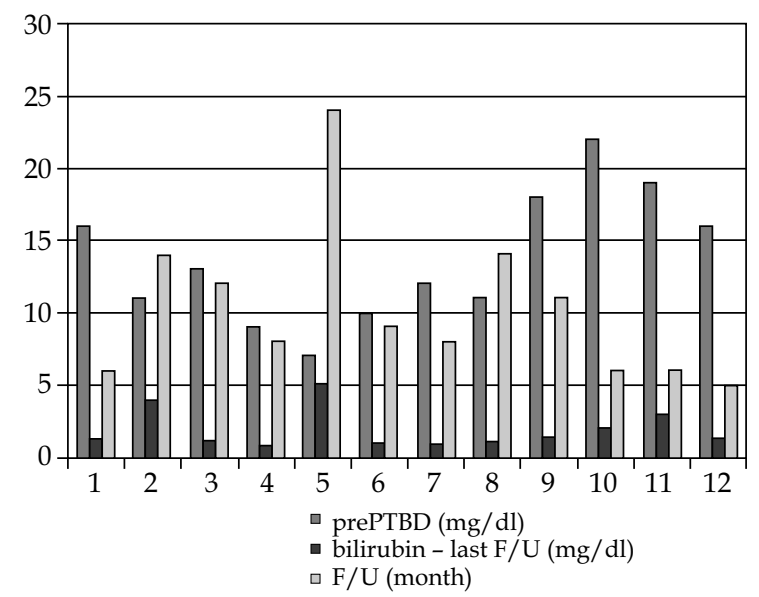

Fig. 3. Bilirubin level - prePTBD, last follow-up and followup duration

patients died with progressive disease and one was lost to follow-up. Stent patency rate of $83 \%$ and stent patency duration of 9.8 months were reported (Figs. 3 and 4).

\section{Discussion}

Diagnosis of malignant obstructive jaundice (MOJ) carries a poor prognosis with one year survival rate of $20-35 \%$ [10]. Most of the patients are diagnosed late with upper abdominal discomfort, general malaise, fever, anorexia, pain, pruritis and jaundice. The intent of treatment is palliation of jaundice, pain, pruritis and associated symptoms. Adequate palliation is achieved once serum bilirubin level is decreased by biliary drainage. Cholangiopancreatography and stenting is the preferred method for proximal/extra hepatic biliary obstruction and PTCD is attempted in distal or terminal biliary obstruction. Often the palliation is curtailed by re-appearance of jaundice, mostly due to stent occlusion. Stent occlusion rates of $30-45 \%$ have been reported in literature [11]. Ingrowth and overgrowth of tumor in the stent has been implicated as the major cause of the stent blockage [1]. Different methods have been tried to prevent stent blockage and growth of disease. Role of radiotherapy in biliary malignancy has been a subject of long debate due to technical problem of delivering high dose without damaging the adjoining critical structures. Many published reports indicated that radiotherapy could provide useful palliation and possibly improve survival in biliary malignancies [12-14]. In 1980 ILBT was introduced for cholangiocarcinoma [6]. The increasing use of PTCD further helped in wider usage of ILBT for MOJ. Many authors have reported their results of ILBT alone or in combination with EBRT $[8,15,16]$. High-dose-rate intraluminal brachytherapy due to its numerous advantages is the preferred choice. Small source size enable itself to be very close to target tissue. Effective therapeutic range of $0.5-2 \mathrm{~cm}$ with rapid fall off of dose from source, thereby minimizing the effect on adjacent normal tissue is another advantage [17]. Using HDR brachytherapy treatment can be accomplished in short time with negligible organ motion and lesser possibility of infection, in contrast with LDR where the applicators and source is kept inside body for several hours to

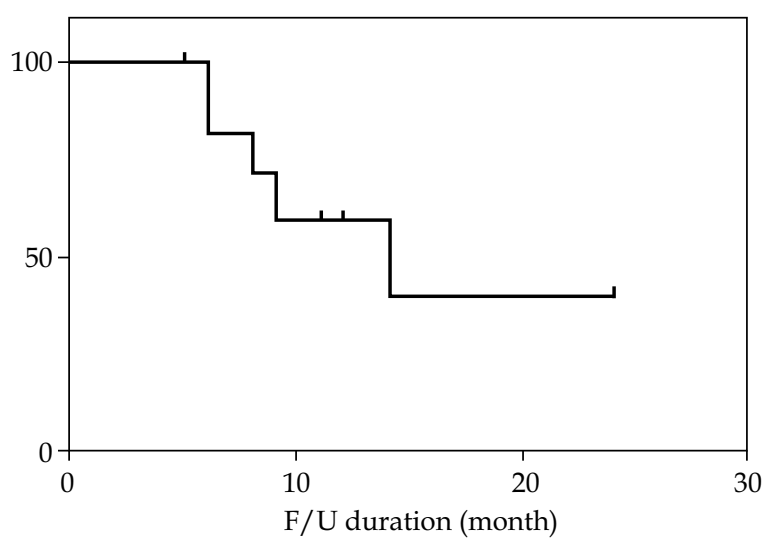

Fig. 4. Survival curve

days. Intraluminal brachytherapy has been reported to be useful in preventing stent occlusion in many centers.

We have used SEMS, because it has been shown that uncovered metallic stents maintain patency longer than plastic stents [18]. We used HDR Ir-192 for ILBT in 12 patients of malignant obstructive jaundice. At a mean follow-up of 10.3 months (5-24), stent patency rates of $83 \%$ and mean stent patency duration of 9.8 months (5-22 months) were observed. All patients tolerated ILBT well with no incidence of perioperative cholangitis, bleeding or shock.

Skowronek et al. [6] reported their experience with pulse dose rate (PDR-ILBT) via PTCD in 48 patients suffering from advanced bile duct and pancreatic cancer. They observed clinical improvement in jaundice in $65.5 \%$ patients with median overall survival time of $\mathbf{1 1 . 2}$ months for bile duct cancer patients. They concluded that PDR-ILBT was feasible, safe and had low complication rate. Dvorak et al. reported their experiences of HDR Ir-192 intraluminal brachytherapy in treatment of 2 groups of patients with MOJ after bile drainage. The causes of MOJ were cholangiocarcinoma and gallbladder carcinoma in one group, cholangiocarcinoma and carcinoma pancreas in the other. The mean survival of both groups was 9 months. After stent placement and subsequent jaundice subsidence, the underlying malignancy could be controlled by ILBT $[19,20]$. Bruha et al. reported patients with MOJ treated with intraluminal brachytherapy using HDR Ir-192. The mean stent patency in patients with cholangiocarcinoma and gallbladder carcinoma were 418 days and 220 days, respectively [21]. Chen et al. showed that the mean stent patency of intraluminal brachytherapy group (12.6 months) was significantly longer than the control group (8.3 months) [22]. Fletcher and colleagues at King's College Hospital analyzed 19 patients, treated with bile drainage and ILBT. Median survival was 11 months, 9 patients survival $>12$ months. They concluded that the addition of internal radiotherapy may be beneficial to patients with hilar cholangiocarcinoma [23]. Montemaggi et al. [24] evaluated ILBT in patients with extrahepatic bile duct or pancreatic cancers. Thirty-one patients received ILBT exclusively or as part of a definitive treatment regimen. ILBT was performed with PTCD in 4 patients and with ERCP in 27 cases. Four- 
teen patients received combined modality treatment: $30 \mathrm{~Gy}$ ILBT and 45 Gy EBRT with continuous infusion of 5-FU. Seventeen patients underwent 50 Gy ILBT alone for palliation. Jaundice was palliated in all patients and pain in 11 out of 13 patients. The survival rate in patients with extrahepatic bile duct cancer was $62 \%$ at second year of combined modality treatment. It was concluded that BT is an effective palliative treatment of unresectable extrahepatic bile duct and pancreatic cancers. Schleicher et al. [25] treated 30 patients of extrahepatic proximal bile duct cancer with schedule consisted of EBRT (median dose $30 \mathrm{~Gy}$ ) and a HDR-ILBT boost (median dose $40 \mathrm{~Gy}$ ) delivered in four or five fractions. Brachytherapy boost dose improved the effect of EBRT by increasing survival from a median of 3.9 months in the non-brachytherapy group to 9.1 months in the brachytherapy group. The effect was obvious in patients receiving brachytherapy dose above $30 \mathrm{~Gy}$.

In our study the mean follow up was 10.3 months (ranged 5-24 months). Two out of twelve patients had stent occlusion at 11 month and 22 month post ILBT. Thus we observed mean stent patency duration of 9.8 months in all patients, resulting in effective and prolong palliation. Mean stent patency durations in cases of cholangiocarcinoma and gall bladder carcinoma were 9.4 months and 10.4 months, respectively. Addition of EBRT and chemotherapy improved stent patency duration to 12.8 months. It might have helped in adequate coverage of target in suitable patients resulting in prolonged stent patency duration.

Johnson et al. [26] found that three out of 11 patients of MOJ treated by EBRT and ILBT developed upper gastrointestinal bleeding from enteritis or frank duodenal ulceration. Since the risk of gastrointestinal bleeding may be dose dependent, therefore we didn't observed bleeding in any patient in our study. Fritz et al. [12] reported the frequency of ulcers in $23 \%$ of patients receiving ILBT doses of 37.5 to $40.0 \mathrm{~Gy}$ and in $7.6 \%$ in those receiving $20.0 \mathrm{~Gy}$. We observed duodenal ulceration in only one patient. We believe that ILBT should be given in most of the cases of malignant stricture induced obstructive jaundice, recurrent stent blockage, cholangiocarcinoma and gall bladder carcinoma invading biliary ducts. It can be given alone or in combination with EBRT. In most cases, effective and prolonged palliation can be achieved, however, an additional systemic trials are required to find appropriate answers for time, dose, fractionation and integration with EBRT.

\section{Conclusion}

Intraluminal brachytherapy after PTCD in treatment of malignant obstructive jaundice is feasible and very effective in preventing stent occlusion with minimal toxicity.

\section{References}

1. Shirabe K, Shimada M, Harimoto $\mathrm{N}$ et al. Intrahepatic cholangiocarcinoma: its mode of spreading and therapeutic modalities. Surgery 2002; 131 (1 Suppl): 159-164.

2. Kim JH, Kim TK, Eun HW et al. Preoperative evaluation of gallbladder carcinoma: efficacy of combined use of MR imaging, MR cholangiography, and contrast-enhanced dual-phase three-dimensional MR angiography. J Magn Reson Imaging 2002; 16: 676-684.
3. Gerbaulet A, Potter R, Mazeron J-J et al. (eds.). The GEC ESTRO Handbook of Brachytherapy. ESTRO, Brussels 2002.

4. Fields JN, Emami B. Carcinoma of the extrahepatic biliary system - results of primary and adjuvant radiotherapy. Int J Radiat Oncol Biol Phys 1987; 13: 331-338.

5. Skowronek J, Sowier A, Skrzywanek P. Intraluminal Pulsed Dose Rate (PDR) brachytherapy and trans-hepatic technique in treatment of locally advanced bile duct cancer - preliminary assessment. Rep Pract Radioth Oncol 2007; 2: 25-33.

6. Skowronek J, Sowier A, Skrzywanek P. Trans-hepatic technique and intraluminal Pulsed Dose Rate (PDR-BT) brachytherapy in treatment of locally advanced bile duct and pancreas cancer. J Contemp Brachytherapy 2009; 2: 97-104.

7. Levitt MD, Laurence BH, Cameron F et al. Transpapillary iridium-192 wire in the treatment of malignant bile duct obstruction. Gut 1988; 29: 149-152.

8. Molt R, Hopfan S, Watson RC et al. Intraluminal radiation therapy in the management of malignant biliary obstruction. Cancer 1986; 57: 536-544.

9. Mornex F, Ardiet JM, Bret E et al. Radiotherapy of high bile duct carcinoma using intracatheter iridium 192 wire. Cancer 1984; 54: 2069-2073.

10. Kawamoto H. Analysis of longterm survivors with expandable metallic stent inserted for malignant biliary stenosis. J Hepatobiliary Pancreat Surg 2003; 1: 95-100.

11. Zhang FJ, Wu PH, Gu YK et al. Clinical value of brachytherapy of malignant biliary obstruction after implanting expandable metallic biliary endoprothesis. Ai Zheng 2004; 23 (11 Suppl): 1567-1571.

12. Fritz E, Brambs H-J, Schraube E et al. Combined external beam radiotherapy and intraluminal high dose rate brachytherapy on bile duct carcinomas. Int J Radiat Oncol Biol Phys 1994; 29: 855-861.

13. Hayes JK, Sapozink MD, Miller FJ. Definitive radiation therapy in bile duct carcinoma. Int J Radiat Oncol Biol Phys 1988; 15: 735-744.

14. Kopelson G, Harisiadis L, Tretter R et al. The role of radiation therapy in cancer of the extrahepatic biliary system: an analysis of 13 patients and a review of the literature of the effectiveness of surgery, chemotherapy and radiotherapy. Int J Radiat Oncol Biol Phys 1977; 2: 883-894.

15. Minsky BD, Wesson MF, Armstrong JG et al. Combined modality therapy of extrahepatic biliary system cancer. Int J Radiat Oncol Biol Phys 1990; 18: 1157-1163.

16. Pilepich MJ, Lambert RM. Radiotherapy of carcinomas of the extrahepatic biliary system. Radiology 1978; 127: 767-770.

17. Manning MA, Arthur DW, Schmidt-Ullrich RK et al. Interstitial high-dose-rate brachytherapy boost: the feasibility and cosmetic outcome of a fractionated outpatient delivery scheme. Int J Radiat Oncol Biol Phys 2000; 48: 1301-1306.

18. Wagner HJ, Knyrim K, Vakil N et al. Plastic endoprostheses versus metal stents in the palliative treatment of malignant hilar biliary obstruction. A prospective and randomized trial. Endoscopy 1993; 25: 213-218.

19. Dvorak J, Petera J, Papik Z et al. Transduodenal intraluminal high dose rate brachytherapy in the treatment of carcinomas of the subhepatic region. Hepatogastroenterology 2002; 49: 10451047.

20. Dvorak J, Jandik P, Melichar B et al. Intraluminal high dose rate brachytherapy in the treatment of bile duct and gallbladder carcinomas. Hepatogastroenterology 2002; 49: 916-917.

21. Bruha R, Petrtyl J, Kubecova M et al. Intraluminal brachytherapy and self expandable stents in nonresectable biliary malignancies - the question of long-term palliation. Hepatogastroenterology 2001; 48: 631-637. 
22. Chen Y, Wang XL, Yan ZP et al. HDR-192Ir intraluminal brachytherapy in treatment of malignant obstructive jaundice. World J Gastroenterol 2004; 23: 3506-3510.

23. Fletcher MS, Brinkley D, Dawson JL et al. Treatment of hilar carcinoma by bile drainage combined with internal radiotherapy using 192 iridium wire. Br J Surg 1983; 12: 733-735.

24. Montemaggi P, Morganti AG, Dobelbower Jr RR et al. Role of intraluminal brachytherapy in extrahepatic bile duct and pancreatic cancers: is it just for palliation? Radiology 1996; 199: 861866.

25. Schleicher UM, Staatz G, Alzen G et al. Combined external beam and intraluminal radiotherapy for irresectable Klatskin tumors. Strahlenther Onkol 2002; 178: 682-687.

26. Johnson DW, Safai C, Goffinet DR. Malignant obstructive jaundice: treatment with external-beam and intracavitary radiotherapy. Int J Radiat Oncol Biol Phys 1985; 11: 411-416. 